\title{
Two finite forms of Watson's quintuple product identity and matrix inversion
}

\author{
X. Ma \\ Department of Mathematics \\ SuZhou University, SuZhou 215006, P.R.China \\ Submitted: Jan 24, 2006; Accepted: May 27, 2006; Published: Jun 12, 2006 \\ Mathematics Subject Classification: 05A10,33D15
}

\begin{abstract}
Recently, Chen-Chu-Gu [4] and Guo-Zeng [6] found independently that Watson's quintuple product identity follows surprisingly from two basic algebraic identities, called finite forms of Watson's quintuple product identity. The present paper shows that both identities are equivalent to two special cases of the $q$-Chu-Vandermonde formula by using the $(f, g)$-inversion.
\end{abstract}

\section{Introduction}

The celebrated Watson's quintuple product identity [5, Exer. 5.7, p. 147] states that for $|q|<1$ and a variable $z$,

$$
\sum_{k=-\infty}^{\infty}\left(z^{2} q^{2 k+1}-1\right) z^{3 k+1} q^{k(3 k+1) / 2}=(q, z, q / z ; q)_{\infty}\left(q z^{2}, q / z^{2} ; q^{2}\right)_{\infty}
$$

where the $q$-shifted factorial $(a ; q)_{n}$ is defined by

$$
(a ; q)_{n}=\prod_{k=1}^{n}\left(1-a q^{k-1}\right), \quad \text { and } \quad(a ; q)_{\infty}=\prod_{k=0}^{\infty}\left(1-a q^{k}\right)
$$

with the following compact multi-parameter notation:

$$
\left(a_{1}, a_{2}, \cdots, a_{m} ; q\right)_{n}=\left(a_{1} ; q\right)_{n}\left(a_{2} ; q\right)_{n} \cdots\left(a_{m} ; q\right)_{n}
$$

This identity has several important applications in combinatorial analysis, number theory and special functions. For its historical note and various proofs, we refer the 
reader to $[2,7]$. Recently, as a new and perhaps the simplest proof, Chen, $\mathrm{Chu}$ and $\mathrm{Gu}[4]$ found that it can be derived simply from the following almost-trivial algebraic identity, called a finite form of the quintuple product identity, the latter is just a limiting case of the terminating $q$-Dixon formula [5, II-14].

Theorem 1 (Finite form of Watson's quintuple product identity) For any integer $n \geq 0$ and a variable $z$, there holds

$$
\sum_{k=0}^{n}\left(1+z q^{k}\right)\left[\begin{array}{l}
n \\
k
\end{array}\right]_{q} \frac{(z ; q)_{n+1}}{\left(z^{2} q^{k} ; q\right)_{n+1}} z^{k} q^{k^{2}} \equiv 1,
$$

where $\left[\begin{array}{l}n \\ k\end{array}\right]_{q}$ denotes the $q$-binomial coefficient $\left[\begin{array}{l}n \\ k\end{array}\right]_{q}=(q ; q)_{n} /\left((q ; q)_{k}(q ; q)_{n-k}\right)$.

Not much later, a somewhat different finite form of this identity was independently discovered by Guo and Zeng [6, Theorem 8.1] via recurrence approach. Their result is

Theorem 2 (Another finite form of Watson's quintuple product identity) For $n \geq 0$ and a variable $z$, there holds

$$
\sum_{k=0}^{n}\left(1-z^{2} q^{2 k+1}\right)\left[\begin{array}{l}
n \\
k
\end{array}\right]_{q} \frac{(z q ; q)_{n}}{\left(z^{2} q^{k+1} ; q\right)_{n+1}} z^{k} q^{k^{2}} \equiv 1 .
$$

For how to derive Watson's quintuple product identity (1.1) from both Eqs. (1.2) and (1.3), we refer the reader to $[4,6]$ for further details. In addition, it is worth mentioning that these two identities are two different $q$-analogues of the binomial relation

$$
\sum_{k=0}^{n}\left(\begin{array}{l}
n \\
k
\end{array}\right) \frac{z^{k}}{(1+z)^{n}} \equiv 1
$$

In this paper, we will show that, with the help of matrix inversion, both Eqs. (1.2) and (1.3) are equivalent to two special cases of the $q$-Chu-Vandermonde formula (cf. [5, II.6])

$$
{ }_{2} \phi_{1}\left[\begin{array}{cc}
q^{-n}, & a \\
& c
\end{array} ; q, q\right]=a^{n} \frac{(c / a ; q)_{n}}{(c ; q)_{n}},
$$

respectively specified by $a=z^{2} q^{n}, c=z q$, and $a=z^{2} q^{n+1}, c=z q$. Here the basic hypergeometric series (cf. $[5, \mathrm{p} .4,(1.2 .22)]$ ) is generally defined by

$$
{ }_{r+1} \phi_{r}\left[\begin{array}{c}
a_{1}, \ldots, a_{r+1} \\
b_{1}, \ldots, b_{r}
\end{array} ; q, z\right]=\sum_{n=0}^{\infty} \frac{\left(a_{1}, \cdots, a_{r+1} ; q\right)_{n}}{\left(q, b_{1}, \cdots, b_{r} ; q\right)_{n}} z^{n}
$$




\section{Matrix inversion}

Our approach is based on an inverse pair of matrices. Let $F=\left(f_{n, k}\right)_{n, k \in \mathbb{Z}}$ be an infinitedimensional lower-triangular matrix over $\mathbb{C}$ subject to $f_{n, k}=0$ unless $n \geq k$. The matrix $G=\left(g_{n, k}\right)_{n, k \in \mathbb{Z}}$ is the inverse matrix of $F$ if

$$
\sum_{n \geq i \geq k} f_{n, i} g_{i, k}=\delta_{n, k} \text { for all } n, k \in \mathbb{Z}
$$

where $\delta$ denotes the usual Kronecker delta. The pair of such two matrices is often called an inversion or a reciprocal relation in the context of combinatorics. As is well known, a fundamental application of matrix inversion is that it provides a standard technique for deriving new summation formulas from known ones. More precisely, assume that $\left(f_{n k}\right)$ and $\left(f_{n k}^{-1}\right)$ are lower-triangular matrices that are inverses of each other, then the following is true:

$$
\sum_{k=0}^{n} f_{n k} a_{k}=b_{n} \quad \text { if and only if } \sum_{k=0}^{n} f_{n k}^{-1} b_{k}=a_{n} .
$$

In $[8,9]$, the author had obtained the following matrix inversion, named the $(f, g)$ inversion.

Lemma 1 Let $f(x, y)$ and $g(x, y)$ be two arbitrary functions over $\mathbb{C}$ in variables $x, y$. Suppose further $g(x, y)$ is antisymmetric, i.e., $g(x, y)=-g(y, x)$. Let $F=(F(n, k))_{n, k \in Z}$ and $G=(G(n, k))_{n, k \in Z}$ be two matrices with entries given by

$$
\begin{aligned}
F(n, k) & =\frac{\prod_{i=k}^{n-1} f\left(x_{i}, b_{k}\right)}{\prod_{i=k+1}^{n} g\left(b_{i}, b_{k}\right)} \quad \text { and } \\
G(n, k) & =\frac{f\left(x_{k}, b_{k}\right)}{f\left(x_{n}, b_{n}\right)} \frac{\prod_{i=k+1}^{n} f\left(x_{i}, b_{n}\right)}{\prod_{i=k}^{n-1} g\left(b_{i}, b_{n}\right)}, \text { respectively, }
\end{aligned}
$$

where $\left\{x_{i}\right\}_{i \in \mathbb{Z}},\left\{b_{i}\right\}_{i \in \mathbb{Z}}$ are arbitrary sequences such that none of the denominators in the right hand sides of (2.2) and (2.3) vanish. Then $F=(F(n, k))_{n, k \in Z}$ and $G=$ $(G(n, k))_{n, k \in Z}$ are a matrix inversion if and only if for all $a, b, c, x \in \mathbb{C}$, there holds that

$$
f(x, a) g(b, c)+f(x, b) g(c, a)+f(x, c) g(a, b)=0 .
$$

As a direct application of Lemma 1, we have the following inverse series relations on the set $\mathbb{N}$ of nonnegative integers instead of $\mathbb{Z}$.

Theorem 3 Let $f(x, y)$ and $g(x, y),\left\{x_{i}\right\}$ and $\left\{b_{i}\right\}$ be given as in Lemma 1. Then for any integers $n \geq 0$, the system of linear relations for any two sequences $\{F(i)\}_{i \in \mathbb{N}}$ and $\{G(i)\}_{i \in \mathbb{N}}$

$$
F(n)=\sum_{k=0}^{n} G(k) f\left(x_{k}, b_{k}\right) \frac{\prod_{i=0}^{k-1} g\left(b_{i}, b_{n}\right)}{\prod_{i=1}^{k} f\left(x_{i}, b_{n}\right)}
$$


is equivalent to the system

$$
G(n)=\sum_{k=0}^{n} F(k) \frac{\prod_{i=1}^{n-1} f\left(x_{i}, b_{k}\right)}{\prod_{i=0, i \neq k}^{n} g\left(b_{i}, b_{k}\right)} .
$$

This special result of the $(f, g)$-inversion will be used in our forthcoming discussion. As stated in our earlier work [9], there are numerous functions forming the $(f, g)$-inversion, i.e., satisfying (2.4). But in what follows, we are only concerned with such a pair of functions $f(x, y)=1-x y, g(x, y)=x-y$. Consequently, we get the special case of Theorem 3 with these parameters $x_{i}=a q^{i}, b_{i}=q^{i}$, which is restated in terms of matrix without proof.

Corollary 1 Let $N(a)$ be an infinite lower-triangular matrix given by

$$
N(a)=\left(\frac{\left(q^{-n} ; q\right)_{k}}{(q ; q)_{k}} \frac{\left(a q^{n} ; q\right)_{k}}{(a q ; q)_{k}} q^{k}\right)
$$

Then the inverse of $N(a)$ is

$$
N^{-1}(a)=\left(\frac{\left(q^{-n} ; q\right)_{k}}{\left(a q^{1+n} ; q\right)_{k}} \frac{(a ; q)_{k}}{(q ; q)_{k}} \frac{1-a q^{2 k}}{1-a} q^{k n}\right)
$$

We remark that this matrix inversion has been used by Andrews in the study of Bailey Lemma. See $[1$, Chapter 3, (3.27)/(3.40)] for the details. As far as we know, it is originally due to Carlitz [3].

\section{Main results}

In this section, we will present our main results. At first, set $f(x, y)=1-x y, g(x, y)=$ $x-y, b_{i}=z q^{i}, x_{i}=z q^{i}$ in Theorem 3. Then an interesting equivalent identity to Eq. (1.2) can be derived via the inverse technique.

Theorem 4 For $n \geq 0$, Eq. (1.2) is equivalent to

$$
z^{n} q^{n^{2}} \frac{1-z}{1-z q^{n}}=\sum_{k=0}^{n}(-1)^{n-k} q^{\left(\begin{array}{c}
n-k \\
2
\end{array}\right)}\left[\begin{array}{l}
n \\
k
\end{array}\right]_{q} \frac{\left(z^{2} q^{n} ; q\right)_{k}}{(z q ; q)_{k}}
$$

or in terms of basic hypergeometric series,

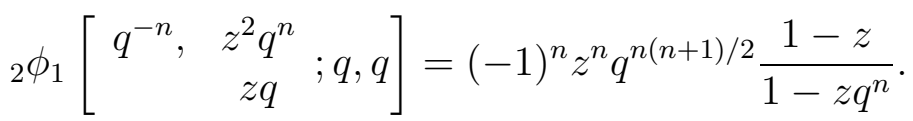


Proof. Assume that Eq. (1.2) holds. Now, using these basic relations

$$
\begin{aligned}
{\left[\begin{array}{l}
n \\
k
\end{array}\right]_{q} } & =\frac{\prod_{i=0}^{k-1}\left(z q^{i}-z q^{n}\right)}{(q ; q)_{k}} z^{-k} q^{-k(k-1) / 2} ; \\
\frac{1}{\left(z^{2} q^{k} ; q\right)_{n+1}} & =\frac{\left(z^{2} ; q\right)_{k}}{\left(z^{2} ; q\right)_{n+1} \prod_{i=1}^{k}\left(1-z^{2} q^{i+n}\right)},
\end{aligned}
$$

we can rewrite Eq. (1.2) as

$$
F(n)=\sum_{k=0}^{n} G(k) f\left(x_{k}, b_{k}\right) \frac{\prod_{i=0}^{k-1} g\left(b_{i}, b_{n}\right)}{\prod_{i=1}^{k} f\left(x_{i}, b_{n}\right)},
$$

where $b_{i}, x_{i}$ are the same as before, and

$$
G(k)=q^{k(k+1) / 2} \frac{\left(z^{2} ; q\right)_{k}}{\left(1-z q^{k}\right)(q ; q)_{k}}, F(n)=\frac{\left(z^{2} ; q\right)_{n+1}}{(z ; q)_{n+1}} .
$$

By the $(1-x y, x-y)$-inversion, we get

$$
G(n)=\sum_{k=0}^{n} F(k) \frac{\prod_{i=1}^{n-1} f\left(x_{i}, b_{k}\right)}{\prod_{i=0, i \neq k}^{n} g\left(b_{i}, b_{k}\right)} .
$$

Inserting the expressions for $G(k)$ and $F(n)$ into (3.4), we deduce immediately that

$$
z^{n} q^{n(n+1) / 2} \frac{\left(z^{2} ; q\right)_{n}}{\left(1-z q^{n}\right)(q ; q)_{n}}=\sum_{k=0}^{n} \frac{\left(z^{2} ; q\right)_{k+1}}{(z ; q)_{k+1}} \frac{\prod_{i=1}^{n-1}\left(1-z^{2} q^{k+i}\right)}{\prod_{i=0, i \neq k}^{n}\left(q^{i}-q^{k}\right)} .
$$

Applying the relation

$$
\prod_{i=0, i \neq k}^{n}\left(q^{i}-q^{k}\right)=(-1)^{n-k} q^{n k-\left(\begin{array}{c}
k+1 \\
2
\end{array}\right)}(q ; q)_{k}(q ; q)_{n-k}
$$

we further reduce $(3.5)$ to

$$
z^{n} q^{n(n+1) / 2} \frac{1-z}{1-z q^{n}}=\sum_{k=0}^{n}(-1)^{n-k} q^{-n k+\left(\begin{array}{c}
k+1 \\
2
\end{array}\right)} \frac{(q ; q)_{n}}{(q ; q)_{k}(q ; q)_{n-k}} \frac{\left(z^{2} q^{n} ; q\right)_{k}}{(z q ; q)_{k}} .
$$

This leads to the desired result Eq. (3.1). Writing this identity in terms of basic hypergeometric series by invoking the relation

$$
(-1)^{k}\left[\begin{array}{l}
n \\
k
\end{array}\right]_{q} q^{\left(\begin{array}{c}
k+1 \\
2
\end{array}\right)-n k}=\frac{\left(q^{-n} ; q\right)_{k}}{(q ; q)_{k}} q^{k},
$$

we get Eq. (3.2).

Conversely, if Eq. (3.1) or Eq. (3.2) is given, then Eq.(1.2) can be also deduced by Theorem 3. This gives the complete proof of theorem.

By the same technique, we derive the following equivalent form of Eq. (1.3). 
Theorem 5 For $n \geq 0$, Eq. (1.3) is equivalent to

$$
z^{n} q^{n^{2}}=\sum_{k=0}^{n}(-1)^{n-k} q^{\left(\begin{array}{c}
n-k \\
2
\end{array}\right)}\left[\begin{array}{l}
n \\
k
\end{array}\right]_{q} \frac{\left(z^{2} q^{n+1} ; q\right)_{k}}{(z q ; q)_{k}}
$$

or in terms of basic hypergeometric series,

$$
{ }_{2} \phi_{1}\left[\begin{array}{cc}
q^{-n}, & z^{2} q^{n+1} \\
z q & ; q, q]=(-1)^{n} z^{n} q^{n(n+1) / 2} .
\end{array}\right.
$$

Proof. The proof is similar to that of Theorem 4, but with the different parameter specification $b_{i}=z q^{i}, x_{i}=z q^{i+1}$. So we omit it.

\section{A bilateral summation}

As pointed out by Chen, Chu and $\mathrm{Gu}$ [4], Eq. (1.2) is just a limiting case of terminating $q$-Dixon formula (cf. [5, II.14]). Thus, it is worthwhile to combine the matrix inversion in Corollary 1 with this classical summation formula in order to find any possibly new or interesting results remained. As an immediate consequence, we obtain the following bilateral summation from which two special cases of Ramanujan's ${ }_{1} \psi_{1}$ summation formula are derived.

Theorem 6 For any integers $m, n \geq 0$, there holds

$$
\begin{aligned}
& \sum_{k=-m}^{n} \frac{\left(q^{-m-n}, x^{2} q^{m-n},-q^{1-n} x / b ; q\right)_{k+m}}{\left(q,-q^{1-n} x, q^{1-2 n} x^{2} / b ; q\right)_{k+m}} q^{k+m} \\
& =\frac{1+q^{-n} x}{1+x q^{m}} \frac{(b ; q)_{m+n}}{\left(q^{1-2 n} x^{2} / b ; q\right)_{m+n}}\left(\frac{-q^{1-n} x}{b}\right)^{m+n} .
\end{aligned}
$$

Proof. Note that the terminating $q$-Dixon formula

$$
{ }_{4} \phi_{3}\left[\begin{array}{cccc}
x^{2}, & q^{-n}, & -q x, & b \\
& q^{1+n} x^{2}, & -x, & q x^{2} / b
\end{array} ; q, \frac{q^{1+n} x}{b}\right]=\frac{\left(q x^{2}, q x / b ; q\right)_{n}}{\left(q x, q x^{2} / b ; q\right)_{n}}
$$

may be reformulated as

$$
\sum_{k=0}^{n} \frac{\left(q^{-n} ; q\right)_{k}}{\left(x^{2} q^{1+n} ; q\right)_{k}} \frac{\left(x^{2} ; q\right)_{k}}{(q ; q)_{k}} \frac{1-x^{2} q^{2 k}}{1-x^{2}} q^{k n} \times \frac{1-x}{1-x q^{k}} \frac{(b ; q)_{k}}{\left(q x^{2} / b ; q\right)_{k}}\left(\frac{q x}{b}\right)^{k}=\frac{\left(q x^{2}, q x / b ; q\right)_{n}}{\left(q x, q x^{2} / b ; q\right)_{n}}
$$

By Corollary 1 specialized with $a=x^{2}$, we get

$$
\sum_{k=0}^{n} \frac{\left(q^{-n} ; q\right)_{k}}{(q ; q)_{k}} \frac{\left(x^{2} q^{n} ; q\right)_{k}}{\left(x^{2} q ; q\right)_{k}} q^{k} \times \frac{\left(q x^{2}, q x / b ; q\right)_{k}}{\left(q x, q x^{2} / b ; q\right)_{k}}=\frac{1-x}{1-x q^{n}} \frac{(b ; q)_{n}}{\left(q x^{2} / b ; q\right)_{n}}\left(\frac{q x}{b}\right)^{n}
$$


After some routine simplification, it reduces to

$$
\sum_{k=0}^{n} \frac{\left(q^{-n} ; q\right)_{k}}{(q ; q)_{k}} \frac{\left(x^{2} q^{n}, q x / b ; q\right)_{k}}{\left(q x, q x^{2} / b ; q\right)_{k}} q^{k}=\frac{1-x}{1-x q^{n}} \frac{(b ; q)_{n}}{\left(q x^{2} / b ; q\right)_{n}}\left(\frac{q x}{b}\right)^{n} .
$$

Making the replacements $n \mapsto m+n, x \mapsto-q^{-n} x, k \mapsto k+m$, we get finally the desired result.

The first case deserving our consideration comes from the case $m=n$ of (4.1), namely

$$
\begin{gathered}
\sum_{k=-n}^{n} \frac{\left(q^{-2 n} ; q\right)_{k+n}}{(q ; q)_{k+n}} \frac{\left(x^{2},-q^{1-n} x / b ; q\right)_{k+n}}{\left(-q^{1-n} x, q^{1-2 n} x^{2} / b ; q\right)_{k+n}} q^{k+n} \\
=\frac{1+q^{-n} x}{1+x q^{n}} \frac{(b ; q)_{2 n}}{\left(q^{1-2 n} x^{2} / b ; q\right)_{2 n}}\left(\frac{-q^{1-n} x}{b}\right)^{2 n}
\end{gathered}
$$

When letting $n \mapsto \infty$ in (4.4) and then replacing $k$ by $-k$ in the resulting identity, we immediately obtain the following nonterminating series identity.

Corollary 2 For three indeterminate $q, x, b$ with $|q|<1$ and $|b|<|x|^{2}<1$, there holds

$$
\sum_{k=-\infty}^{\infty} \frac{(-1 / x ; q)_{k}}{(-b / x ; q)_{k}} x^{2 k-1}=\frac{(q, b,-1 / x ; q)_{\infty}}{\left(x^{2}, b / x^{2},-b / x ; q\right)_{\infty}} .
$$

Another case of special interest is that $b \mapsto 0$ and $m, n \mapsto \infty$ in (4.1).

Corollary 3 For two indeterminate $q$ and $x$ with $|q|<1$ and $0<|x|<1$, there holds

$$
\sum_{k=-\infty}^{\infty} \frac{q^{k(k-1) / 2}}{x^{k}(-x q ; q)_{k-1}}=\frac{(q ; q)_{\infty}(-1 / x ; q)_{\infty}}{\left(x^{2} ; q\right)_{\infty}}
$$

\section{Acknowledgements}

The author thanks the anonymous referee and W.C.Chu for the very detailed comments.

\section{References}

[1] G. E. Andrews, q-Series: their development and application in Analysis, Number theory, Combinatorics,physics and computer algebra, NSF CBMS Regional Conf.Series, Vol.66, 1986.

[2] L. Carlitz and M.V. Subbarao, A simple proof of the Quintuple Product Identity, Proc. Amer. Math. Soc. 32 (1972), 42-44.

[3] L. Carlitz, Some inverse relations, Duke Math. J. 40 (1973), 893-901. 
[4] W. Y. C. Chen, W. C. Chu, and N. S. S. Gu, Finite form of the quituple product identity, J. Combin. Theory, Ser.A, 113 (2006), 185-187.

[5] G. Gasper and M. Rahman, Basic hypergeometric series (second edition), Encyclopedia Math. Appl., Vol.96, Cambridge Univ. Press, Cambridge, 2004.

[6] V. J. W. Guo and J. Zeng, Short proofs of summation and transformation formulas for basic hypergeometric series, J. Math. Anal. Appl., to appear.

[7] H. M. Farkas, I. Kra, On the quintuple product identity, Proc. Amer. Math. Soc. 127 (1999), 771-778.

[8] X. Ma, An extension of Warnaar's matrix inversion, Proc. Amer. Math. Soc. 133 (2005), 3179-3189.

[9] X. Ma, The $(f, g)$-inversion formula and its applications: the $(f, g)$-summation formula, Advances in Appl. Math.(2006), to appear. 